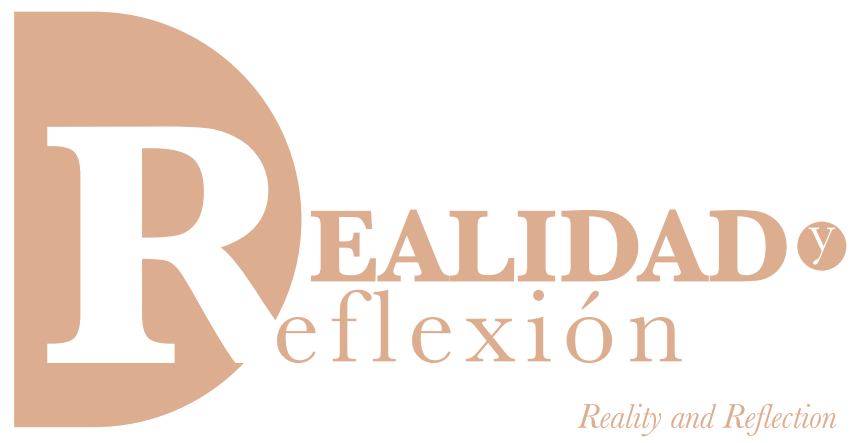

ISSN 1992-6510

e-ISSN 2520-9299

Año 17, N 46, San Salvador, El Salvador, Centroamérica. Revista Semestral Julio-Diciembre 2017

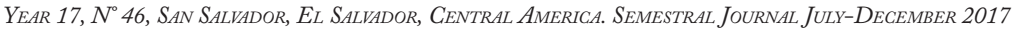

\title{
Una vista a la innovación tecnológica en Centroamérica y América Latina
}

\author{
View of the technological innovation in Central \\ America and Latin America
}

\author{
Víctor Cuchillac ${ }^{1}$ \\ Ingeniero en Electrónica egresado de la Universidad Don Bosco \\ Estudiante de Doctorado en Gestión Pública y Ciencias Empresariales del Instituto Centroamericano de Administración Pública (ICAP) \\ Investigador del Instituto de Ciencia, Tecnología e Innovación de la Universidad Francisco Gavidia \\ Recibido: 25 de abril \\ Aprobado: 15 de diciembre \\ pág. $96-117$ \\ DOI: http://dx.doi.org/10.5377/ryr.v0i46.5511
}

\section{RESUMEN}

Este artículo tiene como objetivo iniciar y promover el diálogo sobre la innovación tecnológica como una estrategia de desarrollo en la economía salvadoreña, porque existen muy pocos indicadores de innovación en el país y la aplicación de la misma como parte de las actividades de las empresas privadas y organizaciones públicas es muy lacónica al compararse con economías en donde la innovación es parte de los procesos productivos y culturales. La evidencia empírica muestra como algunas economías con escasos recursos naturales han encontrado una mejora significativa al incursionar en la innovación tecnológica, para lo cual desarrollaron una infraestructura y una formación de capital humano efectiva y dinámica.

Actualmente el mundo avanza en el desarrollo de las tecnologías como medios de mejora; sin embargo, América Latina no logra despegar en el desarrollo tecnológico, peor aún, la mayoría de los países en Centroamérica no fomentan la investigación básica, la investigación aplicada y el desarrollo experimental, por lo que el capital humano al incorporarse a la vida productiva no produce un salto de calidad o crea las condiciones de una ventaja competitiva que inicie o mantenga el desarrollo sostenible de la economía. Aun cuando existen recomendaciones de organismos internacionales sobre las rutas o las estrategias para los países en vías de desarrollo, los entes encargados de la formación del capital humano no se articulan para trabajar bajo una meta común, es por ello que aquí se esbozan algunas recomendaciones que permitan definir el rol de cada uno de ellos de manera que la innovación tecnológica se fortalezca y pueda convertirse en un futuro próximo en un sector de desarrollo para el país.

Palabras clave: Innovación, Innovación tecnológica, Actividades de investigación y desarrollo (I+D).

1 (c) Víctor Cuchillac 


\section{ABSTRACT}

This article aims to initiate and promote the dialogue on technological innovation as a development strategy in the Salvadoran economy, because there are few indicators of innovation in the country and the application of it as part of the activities of private companies and public organizations where is very laconic when compared to economies where innovation is part of the productive and cultural processes. The empirical evidence shows how some of economies with no natural resources have found a significant improvement when venturing into technological innovation, for which an infrastructure and an effective and dynamic human capital formation have been developed.

Currently, the world is progressing successfully in the development of technologies as means of improvement, however, Latin America has not been able to take off in technological development, even worse, most of the countries in Central America do not encourage basic research, applied research and the experimental development, so that the human capital when incorporating to the productive life does not produce a leap of quality or create the conditions of a competitive advantage that initiates or maintains the sustainable development of the economy. Even when there are recommendations from international organizations about the routes or strategies for developing countries, the entities in charge of the formation of human capital are not articulated to work under a common goal, that is why here are some recommendations that are outlined Enables the role of each of them so that technological innovation is strengthened and will happen in a near future in a development sector for the country.

Keywords: Innovation, Technological innovation, Research and development $(R \in D)$ activities. 


\section{Introducción}

En economías en donde los recursos naturales son muy escasos, incursionar en el desarrollo la tecnología como sector productivo, por medio de la innovación es una estrategia viable, obviamente esto conlleva a realizar una serie de cambios que abarcan la estructura misma de la economía, una política de Estado y una trasformación de las competencias del capital humano. El concepto economía del conocimiento involucra centrarse en el desarrollo de sólidas y actualizadas competencias para capital humano quien toma un papel preponderante en la producción de bienes y servicios ligados a la tecnología, y un ecosistema que favorece la economía local a partir de la innovación. En consecuencia, es necesario comenzar a abordar el tema de la innovación como manera de mejorar la economía de una manera más frecuente; ya que, en el país, temas como la violencia y la política, son los tópicos cotidianos, que si bien es cierto en contadas veces se presentan propuestas, no se percibe una mejora significativa a lo largo del tiempo.

El paradigma de la investigación en nuestra sociedad y parte de la región sigue siendo percibido como una actividad realizada exclusivamente por los científicos o las grandes empresas con laboratorios especializados, además, es muy común que el concepto de la innovación se vea disgregado de la actividad de investigación, lo cual hace que las empresas privadas, las organizaciones públicas y la sociedad civil no involucren la innovación como parte clave de los procesos que realizan. Sin embargo, es bien conocido que culturas asiáticas realizan innovaciones de manera constante en los procesos productivos y no productivos que realizan.
Debido a todo lo anterior, es necesario definir los conceptos de innovación e innovación tecnológica, luego se realizará una exploración de cómo se encuentra el desarrollo y la productividad de la innovación en el país, pero a falta de indicadores nacionales se hará una exploración en la región centroamericana y en América Latina, con lo cual se tendrá una idea del estatus quo, que permitirá ubicar al país con las tendencias mundiales de la investigación, para finalmente hacer una reflexión sobre los entes que pueden impulsar las competencias en el capital humano de manera que la innovación forme parte de la vida académica, empresarial y social del salvadoreño.

\section{El estado de la Investigación y el Desarrollo en América Latina y Centroamérica}

\subsection{El concepto de innovación}

A veces es común pensar que las actividades de innovación está ligadas únicamente a las tecnologías y a los procesos industriales; sin embargo,la innovación posee un espectro de acción más amplio, el abordar epistemológicamente el concepto de innovación es una tarea en la cual no se pueden omitir los postulados del economista austriaco Joseph Schumpeter, quien en su obra de 1942, Capitalismo, socialismo y democracia, expone que el proceso de innovación de los emprendedores crea una fundamentación sólida para el crecimiento económico sostenible, aun cuando este proceso "destruya" el valor y la posición que las empresas anteriormente hayan logrado. Uno de los conceptos más popularizados fue "La destrucción creativa", en la cual se explican los cambios superfluos y radicales que acompañan a las innovaciones en las empresas, las cuales no 
solo se centran en la creación de nuevos productos y servicios. Por su parte, la CEPAL expone que la innovación se ve materializada cuando se mejoran o crean nuevos productos y procesos, sectores y actividades, impulsando de esta manera la trasformación estructural, que a su vez mejora los estímulos a la innovación, creando de esta manera un círculo virtuoso.

A continuación, se presentan algunas definiciones recopiladas por Jeffrey Orozco sobre la innovación desde una perspectiva económica. (Orozco, 2004):

a. "Las innovaciones son nuevas creaciones con relevancia económica. Ellas pueden ser absolutamente nuevas, pero más a menudo son nuevas combinaciones de elementos existentes Ellas pueden ser absolutamente nuevas, pero más a menudo son nuevas combinaciones de elementos existentes", Charles Edquist, en Suiza, 1997.

b. "Es el resultado de procesos continuos de aprendizaje, búsqueda y exploración, que resultan en nuevos productos, nuevas técnicas, nuevas formas de organización, cambios institucionales y nuevos mercados...”, Bjorn Johnson y BengtÅke Lundvall, en Suecia, 1992.

c. "Las innovaciones son nuevos y mejorados productos y procesos, nuevas formas organizacionales, la aplicación de tecnologías existentes en nuevos campos, el descubrimiento de nuevos recursos y la apertura de nuevos mercados", Jorge Niosi y Paolo Saviotti, en Argentina, 1993. d. "Es una mejor cosa que hacer, o una mejor forma de hacerlo, que incrementan la habilidad de una organización para alcanzar sus metas... ofreciendo un impacto duradero", Webster, 1990.

Al analizar los conceptos anteriores se puede concluir que las innovaciones se producen en dos dimensiones: una tangible, que involucra los elementos físicos como son las materias primas y los productos, y una dimensión intangible, que abarca los servicios, las políticas y los cambios organizacionales. Muchas definiciones exponen que las innovaciones se producen cuando se generan nuevas combinaciones a partir de elementos existentes, los cuales, siguiendo un proceso, una técnica o una vinculación diferente, producen una mejora considerable. Desde un punto de vista completamente epistemológico, lo anterior pone en manifiesto que cualquier modificación que provee una mejora a un producto o proceso es una innovación. Pero, dentro de la perspectiva económica (como lo investigado por Jeffrey Orozco), es válido añadir que la mejora debe estar asociada con un impacto económico y sostenible en el tiempo, inclusive Shumpeter considera que "incursionar dentro de un nuevo mercado o modificarlo, como puede ser la ruptura de un monopolio, se considera innovación” (Ateca Amestoy, 2006, p. 4).

\section{¿Qué es la innovación tecnológica?}

En el Manual de Frascati se define claramente el concepto de la innovación tecnológica: (OCDE, 2002, p. 17):

Las actividades de innovación tecnológica son el conjunto de etapas 
científicas, tecnológicas, organizativas, financieras y comerciales, incluyendo las inversiones en nuevos conocimientos, que llevan o que intentan llevar a la implementación de productos y de procesos nuevos o mejorados. La I+D no es más que una de estas actividades y puede ser llevada a cabo en diferentes fases del proceso de innovación, siendo utilizada no sólo como la fuente de ideas creadoras sino también para resolver los problemas que pueden surgir en cualquier fase hasta su culminación.

En áreas como las Tecnologías de la Información y la Comunicación (TIC), debido a su propia naturaleza y ciclo de vida, la innovación es intrínseca, y muchas veces no se observa todo el accionar de las mejoras que ocurren, ya que a veces se observan muy claramente los aportes en el hardware, y no en el desarrollo de software. Sin embargo, el desarrollo de software tiene relevancia dentro de las actividades de innovación. En el Manual de Frascati, la OCDE ha definido la relevancia que el desarrollo del software posee (OCDE, 2002, p. 48):

El modelo en el que originariamente se basaba el Manual era uno en el que la $\mathrm{I}+\mathrm{D}$ se estructuraba institucionalmente en ciencias naturales e ingeniería, que daban lugar a innovaciones tecnológicas tangibles en el sector industrial primario y secundario. Desde entonces, el desarrollo de software se ha convertido en una importante actividad innovadora intangible con un elevado contenido de I+D. Además, cada vez es más importante el porcentaje de actividades que surgen de las ciencias sociales y las humanidades $\mathrm{y}$, junto con los avances informáticos, dan lugar a innovaciones intangibles en las actividades y productos del sector servicios, con una contribución cada vez mayor de las industrias del sector servicios en el sector empresarial.

Acorde a lo anterior hay que considerar que, para que un proyecto de desarrollo de software pueda clasificarse como I+D su creación debe dar lugar a un progreso científico o técnico y su objetivo debe resolver de forma sistemática una incertidumbre científica o técnica. Es decir, crear una aplicación informática que realice una función de otra manera o una versión mejorada sin un salto tecnológico, no se considera $\mathrm{I}+\mathrm{D}$ propia, sino parte del proceso de vida del proyecto de desarrollo de software.

\subsection{Relación de la inversión en $\mathrm{I}+\mathrm{D}$ en la economía}

La Investigación y el Desarrollo (I+D) tiene lugar en toda la economía, pero tiene ciertas características que la distinguen de la familia más amplia de actividades científicas y de económicas de las que forma parte. "Desde el principio, se pretendía que la OCDE estableciera un conjunto de directrices como marco para la medición de la Ciencia, la Tecnología y la Innovación. Con el tiempo, se han añadido manuales adicionales al marco, como el Manual de Estadísticas de las Patentes de la OCDE (OECD, 2009b) y el Manual Oslo (OECD / EUROSTAT, 2005), que proporciona directrices para la recopilación e interpretación de datos sobre innovación. Hay otros también” (OECD, 2015, p. 25). 
Uno de los problemas relacionados con la innovación que se tienen en la región centroamericana es que el gasto en Investigación y Desarrollo, por parte de las empresas, es muy bajo para la mayoría de los países. Por el contrario, como se puede apreciar en la siguiente gráfica, en los países con economías desarrolladas como la República de Corea (Corea del Sur), el Estado de Japón, la República Popular China, la República Federal de Alemania, los Estados Unidos de América, el Reino de Suecia, y la República de Finlandia, el $60 \%$ o más de los gastos son financiados por las empresas. Mientras para las repúblicas de El
Salvador, Guatemala, Costa Rica y Panamá la incipiente inversión por parte de las empresas es inferior al 20\%, en ausencia de la inversión por parte de las empresas no extraña que sean los gobiernos de dichas naciones, los que invierten entre el 20\% (Guatemala) al 60\% (Costa Rica).

En la categoría otros de la gráfica, intervienen instituciones exteriores y sus aportes no son precisamente del financiamiento de la I+D, Para el Caso de Ecuador, El Salvador y Uruguay si invierte en $\mathrm{I}+\mathrm{D}$ universidades, e instituciones sin fines de lucro.

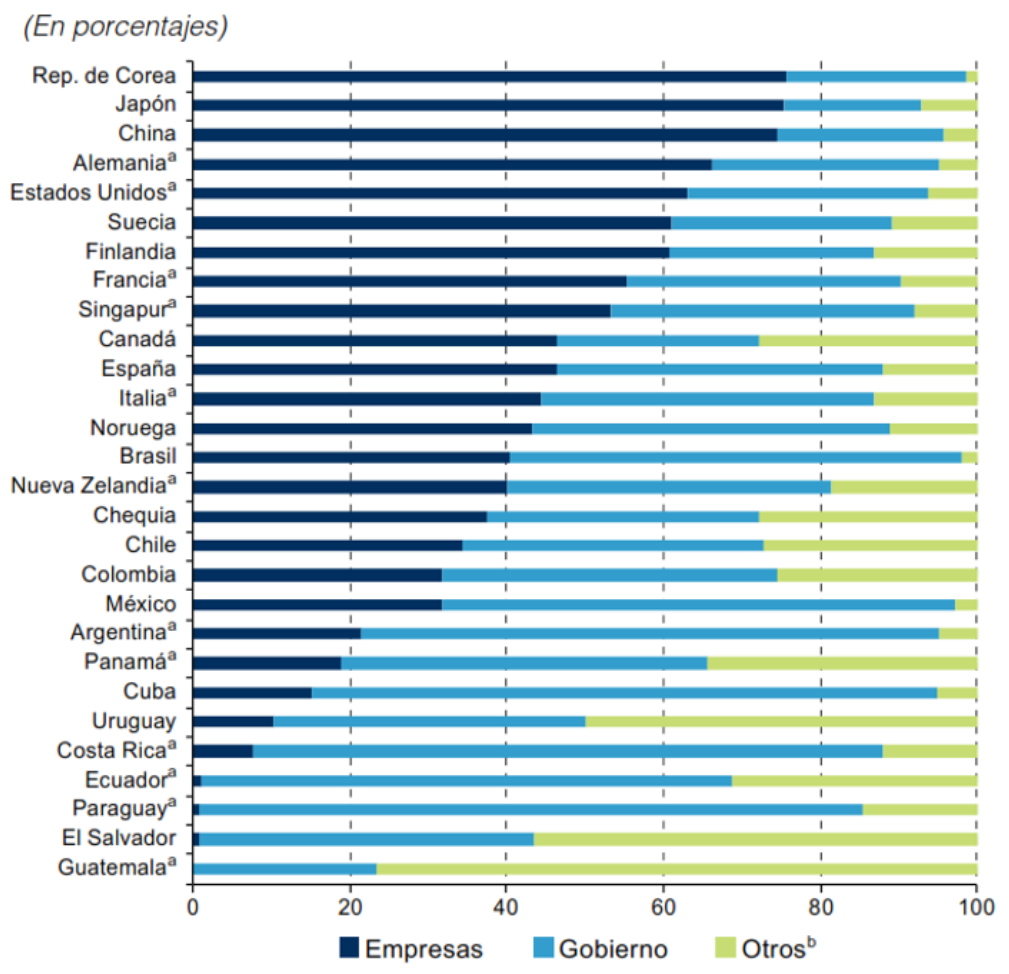

Figura n. 1. El gasto en I+D según el sector de financiamiento en 2012 en porcentajes. Fuente: Comisión Económica para América Latina y el Caribe (CEPAL), sobre la base de información de la Organización de las Naciones Unidas para la Educación, la Ciencia y la Cultura (UNESCO), Organización de Cooperación y Desarrollo Económicos (OCDE) y Red de Indicadores de Ciencia y Tecnología Iberoamericana e Interamericana (RICYT). a. Datos de 2010 o 2011 . b. Se incluye a instituciones de educación superior, organismos extranjeros y entidades privadas sin fines de lucro. 
Como se expuso al inicio, otro indicador de la inversión en $\mathrm{I}+\mathrm{D}$ es la producción de patentes, las empresas al poseer un mayor conocimiento productivo, tienen más y mejores condiciones de crear nuevos productos y servicios, que en la mayoría de los casos son registrados en las diferentes oficinas de patentes. Debido a que los Estados Unidos de América sigue siendo un gran mercado, la oficina de patentes de ese país recibe los registros de muchas innovaciones de casi todo el planeta, por lo cual puede ser un buen "radar" sobre las actividades de I+D globales. En la siguiente gráfica se muestra una comparación de las patentes concedidas a América Latina y otras economías en dónde se aprecia que:

- Los países con economías emergentes de Asia, (en este caso solo se muestra
Malasia, pero Singapur, Corea del Sur $y$ otros mantienen el mismo comportamiento), han tenido un fuerte incremento en la cantidad de patentes que registran, lo cual no es de extrañar si se listan los sectores tecnológicos con los que trabajan: telecomunicaciones, electrodomésticos, automotores, equipo hospitalario, nanotecnología, etc.

- El registro en conjunto de toda América Latina es muy inferior al número de patentes de algunos países. Por ejemplo, a la República de Polonia y la República Popular de China superan año con año la cantidad de patentes de América Latina.

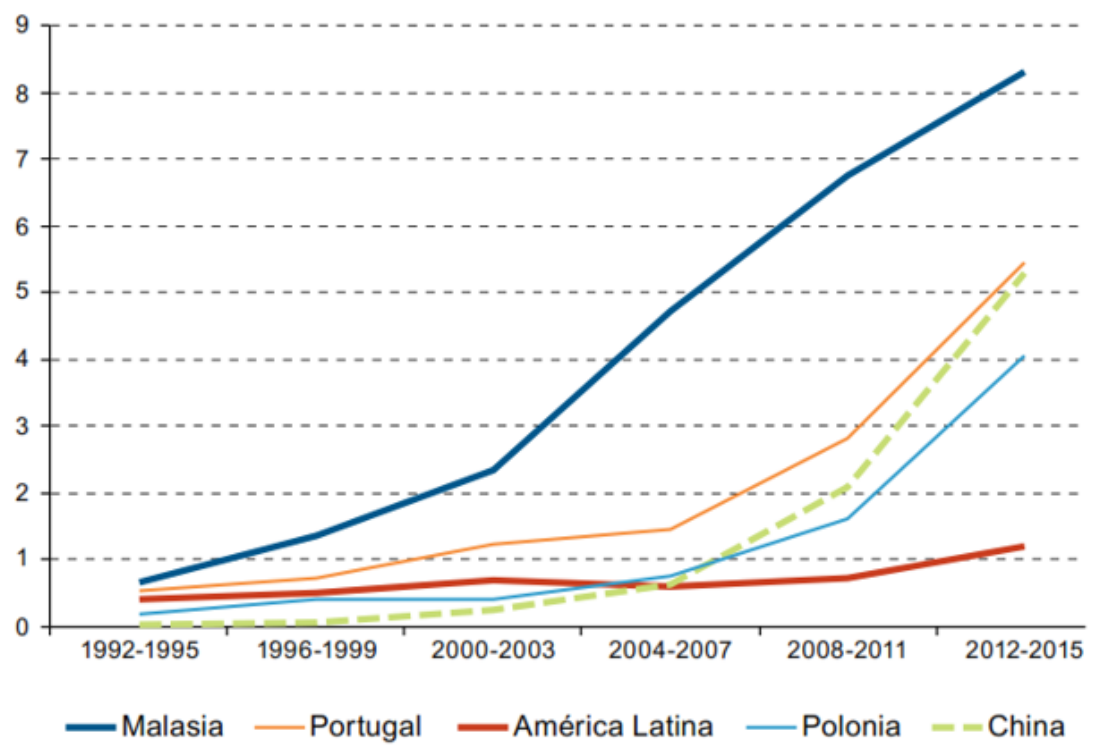

Figura.$^{\circ}$ 2. Patentes concedidas por la Oficina de Patentes y Marcas de los Estados Unidos desde 1992 a 2015 (en número de patentes por millón de habitantes). Fuente: Comisión Económica para América Latina y el Caribe (CEPAL), sobre la base de datos de la Oficina de Patentes y Marcas de los Estados Unidos (USPTO) y Banco Mundial. 
$\mathrm{Al}$ observar en el Cuadro n. ${ }^{\circ} 1$ de los porcentajes de patentes concedidas por la oficina de patentes en Estados Unidos, se observa que América Latina ha incrementado del $0.4 \%$ en 2005, a $0.5 \%$ en 2015 , lo cual es un indicador muy pequeño; es decir, que prácticamente se ha mantenido estancada la producción de patentes durante más de 10 años.

\section{Cuadro n. ${ }^{\circ} 1$}

Distribución del numero de patentes concedidas por la Oficina de Patentes y Marcas de los Estados Unidos a no residentes, para el periodo 2002-2005 y 2012-2015 (en porcentajes)

\begin{tabular}{|l|l|l|}
\hline Países & $\mathbf{2 0 0 2 - 2 0 0 5}$ & $\mathbf{2 0 1 2 - 2 0 1 5}$ \\
\hline Japón & 42.1 & 34.2 \\
\hline Alemania & 13.3 & 10.5 \\
\hline China Taiwán & 7.9 & 7.6 \\
\hline República de Corea & 5.1 & 10.7 \\
\hline Reino Unido & 4.6 & 4.2 \\
\hline Francia & 4.5 & 4.2 \\
\hline Canadá & 4.4 & 4.6 \\
\hline América Latina & 0.4 & 0.5 \\
\hline India & 0.4 & 1.7 \\
\hline
\end{tabular}

¿La innovación tecnológica puede ayudar a mejorar la economía en América Latina?

Al comparar América Latina con algunos países de Asia a mediados del siglo pasado, se observa que los primeros tenían mejores condiciones económicas que los segundos; sin embargo, en la segunda década del presente siglo la diferencia entre algunas economías asiáticas y los países de América Latina presentan una gran diferencia, uno de los grandes factores de esta diferencia consiste en la producción y exportación de tecnologías.

Al analizar la siguiente gráfica, para América Latina y un grupo de economías de Asia, se muestra la relación existente entre la intensidad tecnológica de las exportaciones (medida por el porcentaje de las exportaciones de alta tecnología en las exportaciones totales, en el eje horizontal) y el dinamismo de la demanda por las exportaciones (medido por la participación de las exportaciones de esas agrupaciones de países en las exportaciones mundiales, en el eje vertical). (CEPAL (1), 2016, p. 19). La modificación de su patrón de especialización plantea la CEPAL, fue lo que ha permitido a Asia participar de una forma dinámica y sostenida en el comercio internacional, logrando con ello un crecimiento económico y una mayor cantidad de empleos. Por el contrario, en América Latina no existió un proceso análogo y como consecuencia se tiene una tasa de participación muy baja en las exportaciones mundiales. 


\section{(En porcentajes)}

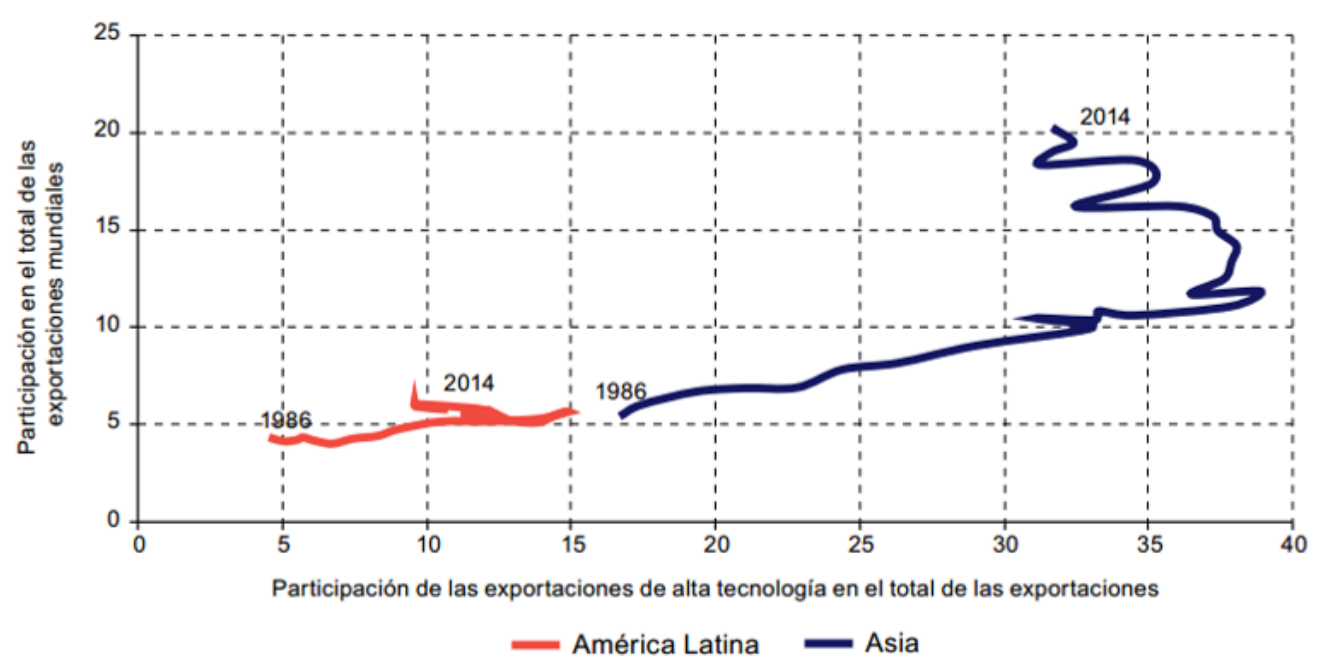

Figura n. ${ }^{\circ}$. Relación entre el nivel de especialización en exportaciones de alta tecnología y la participación en el marcado mundial 1986 - 2014 para América Latina y Asia. Fuente: CEPAL sobre la base de cifras de la Organización Mundial del Comercio (OMC) y Base de Datos estadísticos de las Naciones Unidas sobre el Comercio de Productos Básicos (COMTRADE). a. Se incluyen China, Filipinas, Malasia, la República de Corea, Singapur y Tailandia.

Es necesario exponer también que si las exportaciones aumentan y por ello se produce un mayor ingreso en las economías, no significa que las poblaciones obtienen todos los beneficios de ese ingreso de capital. Al analizar la desigualdad de la distribución de los ingresos de capital, se observa que desde 1980 hasta la fecha ha existido un incremento en el coeficiente de Gini. El coeficiente de Gini es un instrumento estadístico cuya función es cuantificar la desigualdad de los ingresos percibidos en un país por medio de un valor que oscila entre 0 y 1 , siendo 0 el valor utópico en donde los ingresos se distribuyen de forma equitativa entre toda la población y el valor 1 , el cual representaría que solo una entidad recibe todos los ingresos.

Aun cuando se han implementado estrategias y recomendaciones para mejorar la calidad de vida de las personas, la distribución de la riqueza mantiene una leve pero ascendente crecimiento en términos de distribución de la riqueza. Acerca de esto Las Naciones Unidas han realizado un seguimiento en donde se compara el decil más pobre y el coeficiente de Gini de los países de OCDE.

"El coeficiente de Gini de los países de la Organización de Cooperación y Desarrollo Económicos (OCDE) aumentó de 0.29 en los años ochenta a 0.32 en 2013, y esta tendencia se observa tanto en países desarrollados que tradicionalmente han tenido mayores niveles de desigualdad (como los Estados Unidos, cuyo coeficiente aumentó de 0.34 en 1985 a 0.39 en 2013), como en países con una fuerte tradición igualitaria, como los escandinavos" (CEPAL (1), 2016, p. 19). 


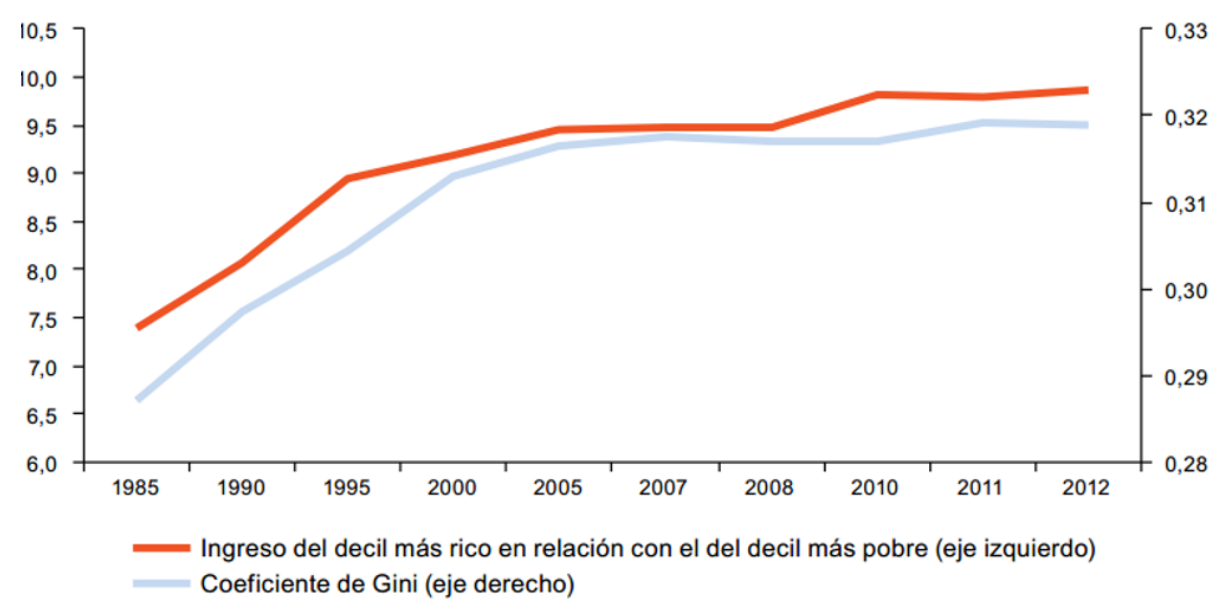

Figura n. ${ }^{\circ}$. Gráfica de la evolución del coeficiente de Gini y la relación entre el ingreso promedio del decil más rico y del decil más pobre para los países de la OCDE. Fuente: CEPAL sobre la base de la OCDE, en It Together: Why Less Inequality Benefits All, París, 2015.

$\mathrm{Al}$ analizar la distribución de la riqueza para la región de América Latina, la CEPAL muestra que la desigualdad aumentó para casi todos los países. En la siguiente figura se muestran las naciones en donde aumentó la desigualdad; es decir, que tuvieron un incremento en el coeficiente de Gini; mientras que, los países debajo de la línea punteada de $45^{\circ}$ son los países en donde hubo un decremento en la desigualdad, y es en esta sección del gráfico que se ubican muchas de las economías de América Latina. Aunque la misma CEPAL aclara que son países en "donde los niveles de desigualdad eran inicialmente $-\mathrm{y}$ siguen siendo- de los más altos del mundo". (CEPAL (1), 2016, p. 20).

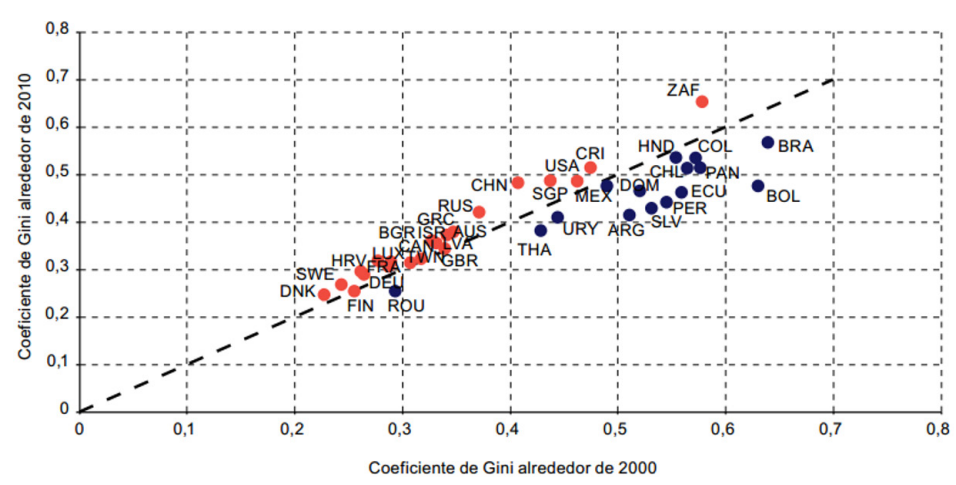

Figura . $^{\circ}$ 5. Coeficiente de Gini para 14 países de A.L y otros países seleccionados durante el período 2000 - 2010. Fuente: Comisión Económica para América Latina y el Caribe (CEPAL), sobre la base de Banco Mundial, World Development Indicators, 2015 y All the Ginis Dataset, Organización de Cooperación y Desarrollo Económicos (OCDE) y Luxembourg Income Study Database (LIS). 


\subsection{Tendencias mundiales de las actividades de investigación}

Para determinar el estado de las actividades relacionadas con la Investigación y el desarrollo para la región Centroamericana, es necesario evaluar qué tipo de estas actividades se están desarrollando tanto en los países avanzados como en los países de la región Centroamericana, para luego compararlas y establecer de manera empírica un nivel del estado. Acorde a las definiciones del manual de Frascati existen tres tipos de actividades (OECD, 2015, pág. 29):

- La investigación básica "es un trabajo experimental o teórico emprendido principalmente para adquirir nuevos conocimientos sobre el fundamento subyacente de fenómenos y hechos observables, sin ninguna aplicación o uso particular a la vista". Los resultados de la investigación básica generalmente no se comercializan, sino que son publicados o divulgados en revistas científicas o actividades científicas.

- La investigación aplicada "es una investigación original emprendida para adquirir nuevos conocimientos. Sin embargo, está dirigido principalmente hacia un objetivo u objetivo específico y práctico”. Este tipo de investigación utiliza los conocimientos disponibles, utiliza el estado de la técnica, o el estado de la ciencia. Los resultados generalmente son patentados o guardados con extremo sigilo por las empresas.

- El desarrollo experimental "es un trabajo sistemático, basado en el conocimiento adquirido de la investigación y la experiencia práctica y produciendo conocimientos adicionales, que se dirige a la producción de nuevos productos o procesos para la mejora de productos o procesos existentes”. Aquí se cuenta con mejores materiales, modificaciones en hardware, software, procesos industriales.

En la siguiente gráfica se detalla el quehacer de las actividades I + D, en donde se puede observar que países como la República Popular de China, el Estado de Israel, los Estados Unidos de América, la República de Corea (Corea del Sur), y el Estado de Japón realizan más del 60\% de "Desarrollo experimental", mientras que los países de Centroamérica cerca del 50\% al 60\% de las actividades corresponde a la investigación aplicada. Es por ello que se infiere que las economías de América Latina continúan importando y aplicando los productos elaborados por las empresas provenientes de los países más desarrollados. Aun cuando en El Salvador y Guatemala se hagan investigaciones básicas, generalmente no se logran darles un uso práctico. Como es común para Centroamérica, muchas de estas investigaciones provienen de Instituciones de Educación Superior, en donde continuando con una agenda relacionada con la generación indicadores (artículos, patentes, etc.), una vez finalizada la investigación básica, se inicia otra y no se le da el seguimiento hasta llevar a otro nivel el trabajo previo. 


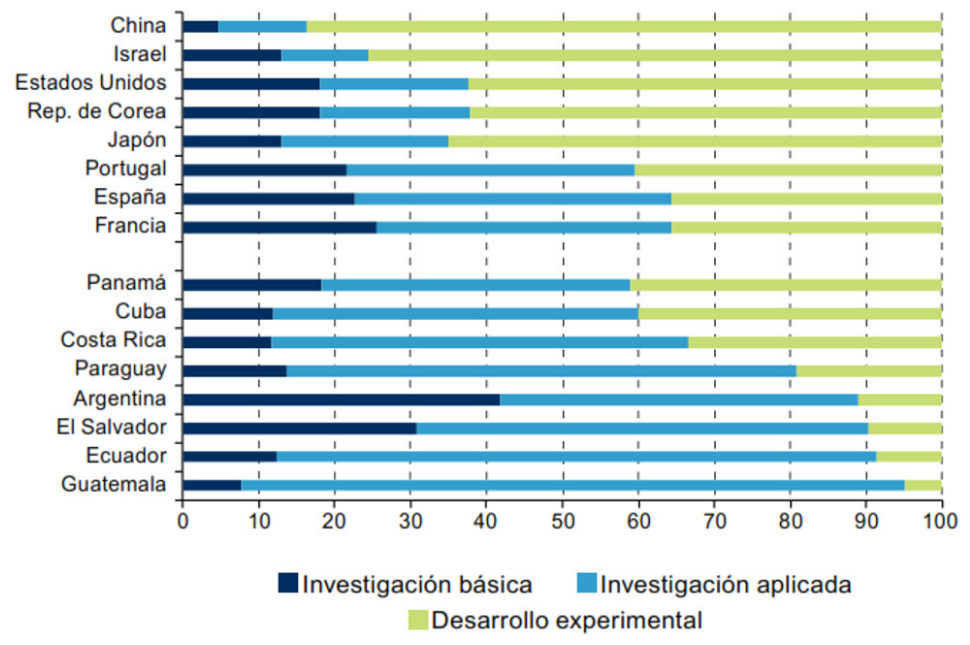

Figura n. ${ }^{\circ}$ 6. Gasto en investigación y desarrollo (I+D) dedicado al desarrollo experimental, promedio de 2010-2013 en porcentajes para países seleccionados. Fuente: CEPAL sobre la base de información de UNESCO y Red de Indicadores de Ciencia y Tecnología Iberoamericana e Interamericana (RICYT).

Uno de los problemas para analizar las acciones de investigación en las economías de Centroamérica es que no todos los países cuentan con un sistema robusto de indicadores, el caso más documentado es Costa Rica, en donde, por ejemplo, se puede observar en el informe del Subsistema Nacional de Indicadores de Ciencia, Tecnología e Innovación
Costarricense que la inversión efectivamente es llevada a cabo por las Instituciones de Educación Superior. (Subsistema Nacional de Indicadores de Ciencia, Tecnología e Innovación, 2015, p. 30). En la siguiente figura se muestra el detalle de inversión en actividades científicas por sector para Costa Rica durante 2010 a 2013.

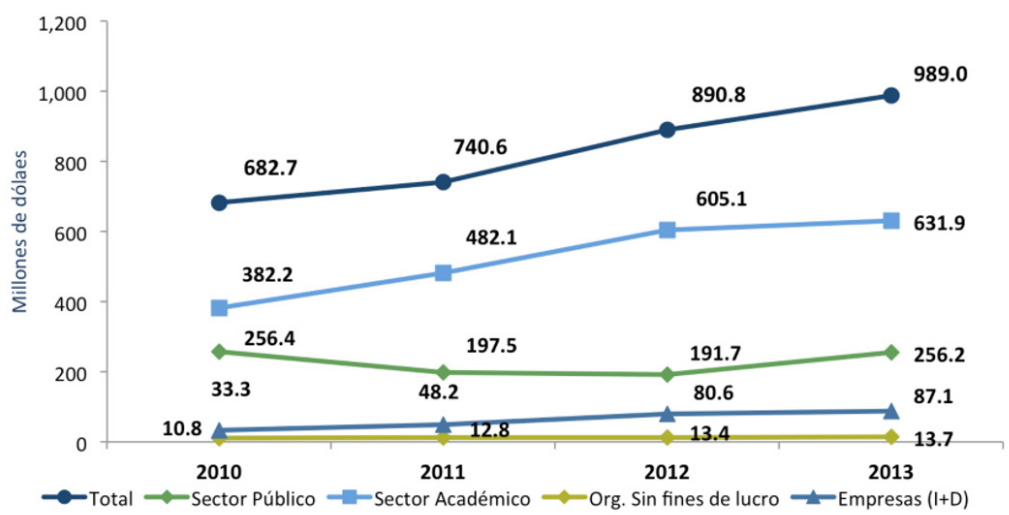

Figura n. 7. Inversión en actividades científicas y tecnológicas según sector de ejecución. 2008-2012-millones de dólares. Fuente: Indicadores Nacionales, Ciencia, Tecnología e Innovación Costa Rica2013, preparado por el Subsistema Nacional de Indicadores de Ciencia, Tecnología e Innovación. 


\section{Algunas recomendaciones de la CEPAL sobre la $\mathrm{I}+\mathrm{D}$ para el desarrollo de $\mathrm{AL}$}

Debido a las condiciones económicas y calidad de vida que imperan en el mundo, muchas organizaciones intentan proponer un plan o un conjunto de estrategias que les permitan mejorar las economías y la calidad de vida de sus habitantes. Por ejemplo, en un estudio reciente realizado por la CEPAL se proponen entre otras, las siguientes estrategias o acciones:

\section{La estrategia para el desarrollo de América} Latina y el Caribe requiere un gran impulso ambiental basado en la difusión de la tecnología

Aunque no es muy clara la fundamentación epistemológica sobre el keynesianismo medioambiental, dicho pensamiento aborda que la inversión del gasto público en proyectos medioambientales "verdes", puede ayudar significativamente a salir de la recesión a las economías en desarrollo y reducir el deterioro del planeta. La CEPAL ve viable este tipo de inversiones "para salir de la trampa del bajo crecimiento y la baja productividad...", (CEPAL, 2016, p. 14), obviamente, esto requiere de proyectos y políticas que deriven en inversiones que fomenten la diversificación productiva y la absorción de tecnología.

\section{Se debe avanzar hacia estructuras productivas más complejas basadas en la innovación}

Los países con economías en desarrollo que solo cuentan con estructuras productivas basadas en la industria tradicional y la agricultura, poseen menos oportunidades de desarrollo que las economías de los países del primer mundo, en los cuales se posee un "Conocimiento productivo", definido como el cúmulo de los conocimientos técnicos y científicos de los productos que se elaboran. Es así que, un país que elabora muchos productos y servicios especializados, posee un mayor nivel de conocimiento productivo que una economía que produce solo materias primas para la exportación e importa tecnología y productos terminados desarrollar las actividades productivas.

Al tener más conocimiento productivo, el capital humano es más preparado e inclusive existe más oportunidad de trabajo para los diferentes niveles de formación. Obviamente, este conocimiento varía para cada país y dependiendo de la especialización o la innovación desarrollada puede incrementar o disminuir su valor en el tiempo, en la siguiente figura se muestra el Índice de Complejidad Económica (ECI), en dónde se muestra el cúmulo de conocimiento presente en la estructura productiva de los países analizados en el decenio de 1995 a 2014.

\section{Las estructuras complejas se vinculan con una mayor inversión en investigación y desarrollo $(\mathrm{I}+\mathrm{D})$ y patentamiento}

La CEPAL hace hincapié en la necesidad de invertir más en actividades de Investigación y Desarrollo (I+D), las cuales pueden ser cuantificadas por el número de patentes, sus publicaciones, el porcentaje del PIB en actividades de $\mathrm{I}+\mathrm{D}$, entre otros. Al buscar los indicadores sobre la inversión en $\mathrm{I}+\mathrm{D}$ para Centro América, se observa que Costa Rica es un país con mejores indicadores. 


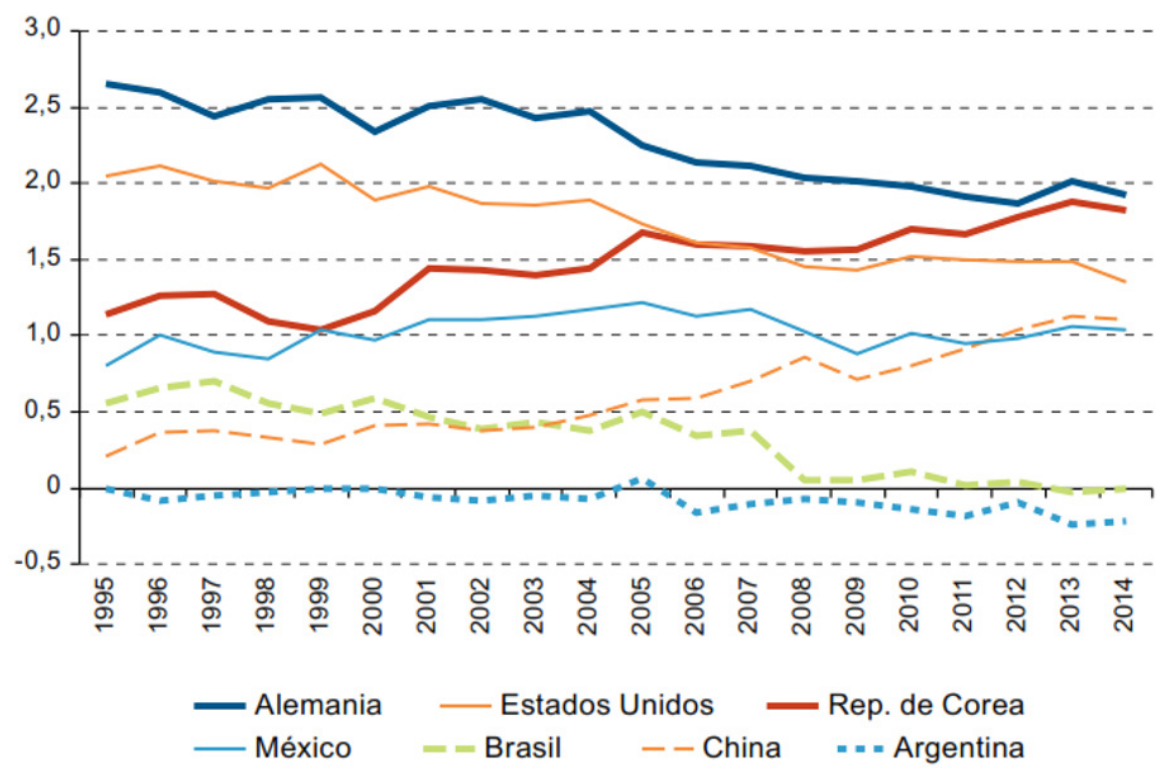

Figura n. ${ }^{\circ}$ 8. Índice de complejidad económica de Hidalgo-Hausmann para período 1995-2014. Fuente: CEPAL, Horizontes 2030: la igualdad e n el centro del desarrollo sostenible (LC/G.2660/ Rev.1), Santiago, 2016.

\begin{tabular}{|c|c|c|c|c|c|c|c|c|c|c|c|c|}
\hline Pais/Indicador & $\begin{array}{l}\text { Costa } \\
\text { Rica }\end{array}$ & Panamá & $\begin{array}{c}E \\
\text { Salvador }\end{array}$ & $\begin{array}{l}\text { Guate- } \\
\text { mala }\end{array}$ & $\begin{array}{l}\text { Hondu- } \\
\text { ras }\end{array}$ & $\begin{array}{l}\text { Nica- } \\
\text { ragua }\end{array}$ & Brasil & Chile & México & $\begin{array}{l}\text { Estados } \\
\text { Unidos }\end{array}$ & Suecia & $\begin{array}{l}\text { República } \\
\text { de Corea }\end{array}$ \\
\hline $\begin{array}{l}\text { Titulados de grado en CT " por } \\
1000 \text { habitantes. }\end{array}$ & 7,5 & 4,2 & 1,4 & 0,6 & 1,2 & 1,2 & 4,1 & 3,4 & 3,2 & 5,1 & n.d. & n.d. \\
\hline $\begin{array}{l}\text { Personal de } \mathrm{CT}^{\circ} \text { por } 1000 \\
\text { habitantes }\end{array}$ & 4,2 & 4,45 & 0,1 & 0,1 & 0,3 & 0,2 & 2,1 & 1,8 & 0,6 & 4,7 & 5 & 4,9 \\
\hline $\begin{array}{l}\text { Gasto en actividades de } 1+D \\
\quad(\% \text { PIB })\end{array}$ & 0,53 & 0,2 & 0,1 & 0,06 & 0,06 & 0,05 & 1,09 & 0,67 & 0,46 & 3,1 & 3,6 & 3,4 \\
\hline $\begin{array}{l}\text { Solicitud de patentes por } \\
\text { residentes por millón de } \\
\text { habitantes }\end{array}$ & 5,5 & 4,5 & 6,5 & 0,4 & 0,7 & 0,5 & 38,2 & 31,6 & 6,4 & 801,8 & 244 & $2.745,9$ \\
\hline $\begin{array}{l}\text { Solicitud de patentes por no } \\
\text { residentes por millón de } \\
\text { habitantes }\end{array}$ & 170,5 & 70,9 & 38,7 & 22,5 & 13,1 & 12,6 & 39,5 & 202,4 & 148,9 & 713,6 & 39,2 & 797,8 \\
\hline $\begin{array}{l}\text { Publicaciones cientificas por } \\
\text { millón de habitantes }\end{array}$ & 97,9 & 107 & 4 & 7,2 & 5,6 & 10,9 & 168,3 & 253 & 90,3 & $1.276,7$ & $1.053,1$ & 464 \\
\hline
\end{tabular}

Figura n.o 9. Indicadores de las capacidades tecnológicas para ciertos países. Fuente: Elaboración a partir de las bases de datos obtenidos del Banco Mundial (World Development Indicators, WDI) (2009), de RICYT y del Anuario Estadístico de América Latina y el Caribe (CEPAL), disponibles en línea.

\section{Hay una relación positiva y significativa entre el ingreso por habitante y la inversión en innovación}

Expertos de la CEPAL exponen que se genera un círculo virtuoso cuando los países crean nuevos productos, servicios y formas de organizar la producción; cambiando así tanto cualitativamente como cuantitativamente las estructuras económicas y sociales, lo que repercute en un aumento del ingreso por habitante, 
así ellos, consumen los nuevos productos y servicios, haciendo que las empresas inviertan nuevamente en la innovación.

Ahora bien, estas recomendaciones no pueden ser implementadas de la misma manera, por todos los países de la región centroamericana; ya que, existen diferencias en las condiciones tecnológicas, el nivel formativo del capital humano y las políticas gubernamentales. Es por ello que, la incursión en la tecnología por parte de los países, deberá ser realizada acorde a las fases más convenientes del ciclo de las tecnologías. Carlota Pérez recopila y expone como "las diferentes tecnologías poseen un ciclo de vida con elementos comunes, en donde cada fase presenta ventajas y oportunidades a los países para que desean incursionar en esa tecnología" (Pérez, 2001, p. 119), Cada tecnología (las tecnologías digitales, la nanotecnología, bioeconomía, etc.), poseen niveles de conocimiento, experiencia en el know how, competencias de la mano de obra no calificada, y como se muestra en la siguiente figura, existen fases en donde el requerimiento de conocimiento científico es muy alto, estas fases son generalmente en países en donde las condiciones de tecnología y conocimiento científico están muy desarrolladas, pero a medida que el ciclo avanza, dicho conocimiento no es tan requerido, y es allí donde países con menores condiciones pueden incursionar, ya que debido a la estandarización de procesos y equipamientos, pueden incorporarse en las fases 2 y 3 , aunque es de señalar que las primeras dos fases son las más rentables. Ejemplo de esto es la industria de los dispositivos inteligentes los cuales son desarrollados en ciertos polos mundiales, como Sillicon Valley, en donde se aglutinan empresas innovadoras y centros de investigación (privados y públicos), pero una vez desarrollados los prototipos, la fase de producción se lleva en países asiáticos como Singapur, Malasia, y China entre otros, debido a las condiciones competitivas de su capital humano menos preparado, los incentivos fiscales de los países, los costos de operación, etc. A continuación, se ilustra el cambio y las oportunidades que presentan las tecnologías acordes a cada fase de evolución.
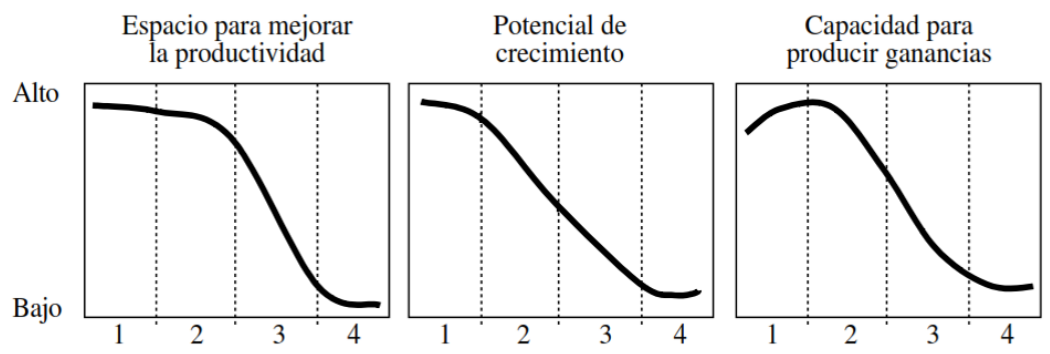

Costo de la inversión (en equipamiento)

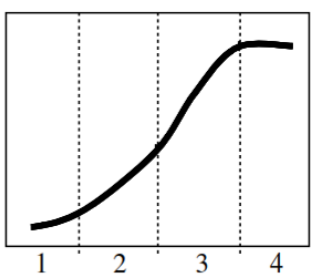

Figura n. ${ }^{\circ}$ 10. Cambio en el potencial de las tecnologías según la fase de evolución de las tecnologías. Fuente: Basado en Gerschenkron (1962), Cundiff y otros (1973), Kotler (1980) y Dosi (1982). 


\section{Roles de los impulsores de la innovación tecnológica en Centroamérica}

\subsection{El rol de las Instituciones de Educación Superior}

Como se expresado con anterioridad, "la innovación no está circunscrita a la tecnología", pero en la innovación tecnológica el uso de esta última es por antonomasia. Es por ello que las IES poseen grandes retos a la hora de formar al capital humano en áreas de innovación tecnológica: Fortalecer las competencias de los catedráticos, vincularse estrechamente con las empresas líderes en innovación tecnológica y contar con laboratorios adecuados para el cambio tecnológico. Es por ello que las IES deben desarrollar un modelo que les permita moverse eficaz y efectivamente en el desarrollo de la Innovación.

Acorde a Nathan Rosenberg, profesor de economía de la Universidad de Stanford, bajo la óptica de la Investigación, el Desarrollo y la Innovación $(\mathrm{I}+\mathrm{D}+\mathrm{i})$, existen dos modelos globales de universidades, las estadounidenses y las europeas, ${ }^{2}$ en las primeras, se destacan los logros económicos por estar insertas en una economía enorme; además, del hecho que desde la postguerra el gobierno federal aportó grandísimas cantidades de dinero para apoyar la actividad científica. Un segundo elemento a considerar es que las universidades estadounidenses han respondido de forma

2 Aunque el autor en su escrito, manifiesta que "no pretendo colocar el sistema universitario estadounidense como un modelo que es seguido al pie de la letra por las universidades", se observa de forma indirecta que el modelo $\mathrm{I}+\mathrm{D}+\mathrm{i}$ de las universidades estadunidenses, es tomado por otras universidades europeas. resuelta ante los cambios de las necesidades económicas y las oportunidades, “...siendo ejemplo de esto, la creación de nuevas ingenierías con orientación práctica, aun cuando otros países han sido líderes científicos", (Briceño Gill, 2014, pp. 28-29), Rosember expone que "la combinación de respuesta y alta calidad de enseñanza e investigación se puede atribuir a ciertos elementos del sistema y a los incentivos que han emanado de ellos", un ejemplo de lo anterior es el sistema de incentivos que existen en las universidades de la costa oeste de los Estados Unidos de América, en donde muchos estudiantes logran estipendios y contratos al desarrollar los proyectos de sus asignaturas o de fin de clico en empresas de tecnología.

Retomando lo anterior, es necesario modificar el sistema actual de las Instituciones de Educación Superior (IES) centroamericanas, en lo relacionado con la formación de las competencias técnicas de sus catedráticos y la vinculación directa con el sector productivo. Porque los catedráticos e instructores no son formados o certificados en las disciplinas o áreas claves de la innovación tecnológica. Esto generalmente por aspectos financieros; ya que, certificar al cuerpo docente involucra un desembolso financiero mucho mayor que los cursos de nivelación o actualización tradicionales. Además, el cuerpo docente una vez capacitado debe ser incluido en un programa de aseguramiento; ya que, con las nuevas competencias adquiridas se tiene el riesgo que pueda migrar del claustro académico a la industria, repercutiendo con ello en una inversión sin retorno para las IES. Es por eso que, desarrollar un sistema basado en escalafones o incentivos, presenta una nueva inversión mucho más onerosa que solo las propias certificaciones. 
Lo complejo de esto es que, si el cuerpo docente no está actualizado técnicamente y no posee las competencias para la innovación tecnológica los estudiantes que formen tendrán debilidades en sus perfiles profesionales, impactado directamente e indirectamente a las empresas que los contraten, porque deberán invertir estas últimas en nivelar el perfil profesional o no recibir el desarrollo innovador que deberían producir los nuevos profesionales.

Lo agravante en formación técnica es el binomio "Docente certificado y equipo actualizado"; ya que, es casi imposible realizar las estrategias metodológicas que logren el desarrollo de las competencias técnicas sin los instrumentos, los equipos y los accesorios para las guías de laboratorio o los proyectos de investigación/innovación. Contar con las condiciones de laboratorios óptimas requiere de una gran inversión, lo cual genera en la IES que deba aumentar la cuota de escolaridad o los aranceles por uso de laboratorios (si no se tiene otra forma de obtener fondos para el equipamiento). Pero el aumentar la cuota presenta un comportamiento inverso proporcional a la demanda de estudiantes. Es por ello, que es común en la región centroamericana que muchas IES solo cuenten con Centros de cómputo como "únicos laboratorios actualizados" para las carreras técnicas.

En las IES los montos de inversión en Investigación, Desarrollo e Innovación son muy paupérrimos, por ejemplo, en El Salvador la Ley de Educación Superior exige que las IES inviertan como mínimo un $2 \%$ del presupuesto anual, los cuales son absorbidos por los salarios, y los costos administrativos del personal con las tareas de investigación. Si la IES desarrolla investigación esta es realizada por los docentes y/o los investigadores, pero no existe fondo para que los estudiantes desarrollen proyectos que terminen en la producción de prototipos que generen patentes, planos industriales, como ocurre en las universidades de la costa oeste de los Estados Unidos de América.

Otro reto para las IES de la región Centroamérica es que no se cuenta con una relación intrínseca con los sectores productivos; es por ello, que se producen planes de estudio alejados de las necesidades actuales y futuras de las empresas, y no existe un mecanismo (o si existe es muy estrecho), de experimentación en situ, es decir, que los estudiantes o catedráticos pueda visitar los centros laborales para conocer más de las tecnologías que se utilizan. Por eso no existe asombro al hecho que muchos profesionales al finalizar sus estudios no tengan experiencia concreta en las competencias claves de los sectores productivos o deban recibir al incorporarse en las empresas los entrenamientos sobre dichas tecnologías.

\subsection{E1 rol de las organizaciones privadas}

Para las empresas ser competitivo nacional, regional o mundialmente cada día, es más difícil, y no existe una garantía que los éxitos pasados en la comercialización de bienes y servicios continúen en el futuro. Thomas Davenport y Laurence Prusack ya lo exponían con el caso de Estados Unidos, al final del siglo pasado:

La demanda de bienes estadounidenses en el país y en el extranjero era tan grande que casi cualquier producto podría encontrar un mercado. Hoy, la participación de los EE.UU. en el PIB 
mundial es de aproximadamente el 18 por ciento. Aunque el pastel es mucho más grande de lo que era, las empresas estadounidenses ya no dominan el mercado mundial. Hay una feroz competencia internacional por cada dólar marginal de ganancias. Una economía rápidamente globalizada, unificada por la mejora de la comunicación y el transporte, ofrece a los consumidores una selección sin precedentes de bienes y servicios $y$ una cabalgata sin fin de nuevas y mejores ofertas de empresas globales. (Davenport \& Prusack, 1998, p. 13).

Esta realidad mundial es más severa en Centroamérica en dónde las empresas dependen en gran medida de la adquisición de las materias primas, los conocimientos y la tecnología de otros países para la producción de los bienes y los servicios que ellas comercializan. Por eso, es muy común que en nuestros países sean las empresas transnacionales las que se encarguen de los servicios de telefonía, la televisión por cable, los proveedores de Internet, las cadenas de hoteles, las productoras de alimentos y medicamentos, entre otros rubros claves. Las empresas de base tecnológica en la región centroamericana se cuentan con los dedos de la mano. Ramón Padilla, oficial de Asuntos Económicos de la Comisión Económica para América Latina y El Caribe (CEPAL), sede subregional en México, asegura que:

En algunos países de Centroamérica, como Honduras y Nicaragua, este concepto es prácticamente inexistente (...) Los Gobiernos de los países que integran la región centroamericana han hecho tímidos esfuerzos por impulsar un sistema de innovación regional, y ninguno ha trascendido" (Forbes Staff, 2017).

La excepción viene siendo Costa Rica, en dónde existen mejores condiciones e infraestructura tecnológica que en el resto de países, para generar más oportunidades de desarrollo tanto a la sociedad como a las empresas.

Es muy conocido el ejemplo de países que han tenido un crecimiento económico asociado al desarrollo tecnológico (entre otros), en especial se puede mencionar el caso de la República de Taiwán y Corea del Sur, en los cuales hasta la mitad de la década de los años 70 se tenían niveles del PIB muy cercanos a los de E1 Salvador y levemente menores al PIB de Costa Rica. Sin embargo, ahora se encuentran con valores muy distantes de los valores del PIB centroamericano, la evidencia empírica propone que los países de rápido crecimiento son economías que basaron su desarrollo en el conocimiento; es decir fomentando la "Economía del conocimiento". En la región Centroamericana el mejor caso es Costa Rica, la cual ha desarrollado múltiples sectores relacionados con la Tecnologías de la Información y Comunicación (TIC) y se ha convertido en proveedor clave de tecnología a otros mercados.

La cultura de investigación es muy baja y casi nula en muchos sectores de la producción del triángulo norte de Centroamérica, porque no se tiene la metodología y los recursos financieros. Es por ello que se depende de la importación de tecnología e insumos para los procesos de producción,yno se puede innovar para darunvalor agregado a los productos que se comercializan. 
También existe una pobre formación en otros idiomas en especial el inglés que es el utilizado por el mayor "socio" económico del triángulo norte de Centroamérica. La organización de las empresas presenta estructuras tradicionales, en las cuales no existen planes de evaluación del desempeño integral de los trabajadores; mecanismos de incentivos salariales y/o sociales; prácticas laborales, como la rotación de puestos, las pasantías, los programas de certificación, el trabajo en equipo, las reuniones "adhoc" que permitan a los empleados tomar decisiones sobre la mejora del servicio, producto, el proceso o la estructura de la empresa.

También en las empresas se observan algunos comportamientos expuestos en las IES, como son la ausencia de sistemas de incentivos, porque también especializar a la mano de obra calificada pone en riego la fuga del personal. La vinculación la IES es endeble, porque trabajar en conjunto con las IES no es parte de la cultura en muchos lugares; ya sea, por miedo a dar a conocer el "know how", por mezquindad o por el desconocimiento de los beneficios en el desarrollo de proyectos mutuos. La relación con las IES en la mayoría de las veces se circunscribe al servicio social que deben realizar los estudiantes; ya que, para las empresas la participación de los estudiantes se convierte en mano de obra muy barata o "gratis".

\subsection{El rol de la gestión gubernamental}

En la mayoría de los países centroamericanos no se desarrollan Planes de Nación ni Políticas Públicas de largo alance, porque los planes y políticas propuestas por los anteriores Gobiernos son descartadas o modificadas por el nuevo Gobierno; ya que, la búsqueda y logro del rédito político al final del período supone que el nuevo Gobierno debe presentar una imagen "redentora" e "innovadora" sin precedentes, en donde los logros y avances del país se deban exclusivamente a la gestión gubernamental actual. También en esa lógica, la gestión gubernamental se mueve por la agenda pública del momento y no por las estrategias a largo plazo.

Debido a las condiciones de inseguridad en el triángulo norte centroamericano y su prioridad en la gestión gubernamental, se producen falencias en las Políticas Públicas, en donde falta de manera estratégica que se desarrollen los planes que permitan enfocarse en el bono demográfico, desarrollar de forma estratégica las zonas de cada país, y proveer la infraestructura para la Banda Ancha a la sociedad. No existe un efectivo fortalecimiento de los centros de investigación nacionales, los nulos fondos para la especialización en el extranjero de los ciudadanos, la ausencia de incentivos fiscales a las empresas por la investigación y no por la asistencia social (el caso de las Teletones). Es por todo lo anterior que mejorar la planificación estratégica para la innovación tecnológica, involucra hacer una sinergia entre el Gobierno nacional, el gobierno local, las IES y las empresas, en donde de forma comprometida se definan los planes y estrategias a corto mediano y largo plazo.

Los Gobiernos de la región deben realizar esfuerzos que permitan aprovechar los cambios tecnológicos de manera que la innovación sea una parte clave en todos los sectores de la sociedad, estos esfuerzos deben ser realizados acorde a la realidad tecnológica de cada país centroamericano, ya que, al contemplar el estado actual de la región se observa que sólo 
Costa Rica posee las mejores condiciones para contar con indicadores de tecnología, y a partir de estos, tomar las medidas o los insumos para la creación de Políticas Públicas. En Costa Rica se creó un organismo desde la década de 1970 (CONICYT) $^{3}$ mientras que el resto de países se hicieron dos décadas después, en la década de 1990. (CONACYT ${ }^{4}, \mathrm{CONCYT}^{5}$, $\mathrm{COHCIT}^{6}, \mathrm{CONICYT}^{7}$ y el $\mathrm{SENACYT}^{8}$ ), es por ello que no es de extrañarse que Costa Rica posea un mayor porcentaje del PIB (más de 0.4\%), destinado a la Investigación y Desarrollo I+D que todos los demás países de la región (por debajo de 0.1\%) (Fernández Polcuch, Bello, \& Massarani, 2016, p. 22). Los gobiernos deben trabajar en dos líneas, la primera en definir Políticas Públicas efectivas que promuevan el desarrollo de la investigación, el desarrollo y, la innovación, en especial el área de las TIC. Y la segunda acción a realizar es dinamizar y apoyar a los Centros de investigación nacionales. Acorde una serie de estudios del Banco Interamericano de Desarrollo BID, William Marroquín expone que, en algunos países centroamericanos tanto la sociedad como el sector económico, no tienen interés por el desarrollo científico y tecnológico. (Marroquín, 2013, pp. 28 - 29).

3 Consejo Nacional de Investigaciones Científicas y Tecnológicas (CONICYT) en Costa Rica.

4 Consejo Nacional de Ciencia y Tecnología (CONACYT) en El Salvador.

5 Consejo Nacional de Ciencia y Tecnología (CONCYT), en Guatemala.

6 Consejo Hondureño de Ciencia y Tecnología (COHCIT), en Honduras.

7 Consejo Nicaragüense de Ciencia y Tecnología (CONICYT), en Nicaragua.

8 Secretaría Nacional de Ciencia y Tecnología (SENACYT), en Panamá.
Un ejemplo de cómo el Gobierno ayuda a las iniciativas de innovación tecnológica es el caso de Paulo Díaz, quien, en un concurso de emprendimiento, ganó un fondo de incubación para su proyecto de investigación universitario lo que le permitió crear The Gluten Free Factory la cual es ahora una firma costarricense con siete años en el mercado, que desarrolla premezclas libres de gluten para pan, pizza, pancakes o waffles y brownies. (Forbes Staff, 2017).

\section{Conclusiones}

La innovación no es un paradigma circunscrito exclusivamente a círculos científicos únicamente, por el contrario, debe ser una actividad que la sociedad salvadoreña debe incorporar en todo su actuar, y aprovechando el constante avance de la tecnología, la innovación tecnológica también debe ser incluida como un eje transversal de desarrollo, con el fin de contribuir en la mejora de los aspectos tangibles como son: las materias primas y los productos terminados, y a los aspectos intangibles como son: los servicios, las estructuras organizacionales, y las políticas públicas entre otros, logrando con ello mejorar el bienestar de la sociedad e impactar significativamente el desempeño de la economía.

De la información mostrada se puede establecer una fuerte correlación entre las economías fuertes y las actividades de investigación, desarrollo e innovación (I+D+i), también de dicha información se puede observar que, tanto en la América Latina como en El Salvador, dichas actividades son muy incipientes, sin embargo, ante este panorama desalentador existe la evidencia empírica que la incursión en desarrollo de las tecnologías como sector productivo 
presenta a los países diferentes oportunidades para mejorarlas sus economías. Acorde a las características de los países centroamericanos, es viable crear políticas públicas y programas de incentivo en las empresas y universidades que permitan desarrollar la cultura de innovación.

Desarrollar las condiciones para que se genere la economía del conocimiento es una acción articulada ente los siguientes actores: el sector empresarial, las instituciones de formación superior y el gobierno, como se ha expuesto cada uno de ellos debe hacer los cambios en sus organizaciones de manera que se incida eficaz y eficientemente en el capital humano y se produzca la infraestructura de servicios para que el país pueda incluirse en una de las fases del ciclo de la tecnología.

\section{Referencias bibliográficas}

Ateca Amestoy, V. (16 de junio de 2006). Economía de la Innovación Tecnológica. Obtenido de Departamento de fundamentos del análisis económico.: http://www.ehu.eus/ vickyateca/notas_clase.pdf

Briceño Gill, M. Á. (2014). Universidad, Sector Productivoy Sustentabilidad. Caracas: Consejo de desarrollo científico y humanistico.

CEPAL (1). (2016). Horizontes 2030. La igualdad en el centro del desarrollo sostenible. Santiago de Chile: Autor.

Comisión Económica para América Latina y el Caribe. (2016). Ciencia, tecnología e innovación en la economía digital, La situación de América Latina y el Caribe. Santiago de Chile: Autor.
Davenport, T. H., \& Prusack, L. (1998). Working Knowledge. How Organizations Manage what they know. Boston: Hardvard Business School Press.

Fernández Polcuch, E., Bello, A., \& Massarani, L. (2016). Politicas públicas e instrumentos para el desarrollo de la cultura cientifica en América Latina. Montevideo: UESCO.

Forbes Staff. (22 de marzo de 2017). ¿A nadie le importa la innovación en Centroamérica? Obtenido de FORBES México: https:// www.forbes.com.mx/a-nadie-le-importa-lainnovacion-en-centroamerica/

Marroquín, W. (2013). Academia. Obtenido de Indicadores de Ciencia y Tecnología: http:// www.academia.edu/8019079/Capacidades_ para_la_recolección_y_análisis_de_ indicadores_de_ciencia_tecnología_e_ innovación_en_los_países_centroamericanos

OCDE. (2002). Manual de Frascati. Propuesta de Norma Práctica para Encuestas. París: Fundación Española para la Ciencia y la Tecnología (FECYT).

OECD. (2015). Frascati Manual 2015: Guidelines for Collecting and Reporting Data on Research and Experimental Development. París: OECD Publishing.

Orozco, J. (2004). Innovation and Performance Improvements in the Cooperative Sector. Dinamarca: CINPE.

Pérez, C. (2001). Cambio tecnológico y oportunidades de desarrollo como blanco móvil. Revista CEPAL (75), 118. 
Subsistema Nacional de Indicadores de Ciencia, Tecnología e Innovación. (2015). Indicadores Nacionales, Ciencia, Tecnología e Innovación Costa Rica. San José: MICITT. 